\title{
Chronic Wasting Disease: Current Assessment of Transmissibility
}

\author{
Akikazu Sakudo ${ }^{1,2 \star}$
}

\begin{abstract}
${ }^{1}$ Laboratory of Biometabolic Chemistry, School of Health Sciences, University of the Ryukyus, Nishihara, Japan.

${ }^{2}$ Faculty of Veterinary Medicine, Okayama University of Science, Imabari, Ehime, Japan.

${ }^{*}$ Correspondence: akikazusakudo@gmail.com
\end{abstract}

https://doi.org/10.21775/cimb.036.013

\begin{abstract}
Chronic wasting disease (CWD) is a prion disease of cervids characterized by clinical symptoms of progressive weight loss, abnormal behaviour and excessive salivation. Incidents have been reported in North America and Korea as well as in Europe. Current knowledge, based on in vitro and in vivo experiments, suggests direct CWD transmission to humans is unlikely. Nonetheless, humans may consume CWD-infected materials, which presents a potential risk. Studies indicate that transmission by horizontal infection of cervids probably occurs via saliva, faeces, and urine as well as from environmental reservoirs of prions found in soil and water. In addition, infectivity in the skeletal muscle of infected deer has been observed. These findings suggest that direct contact with infected animals and indirect contact with prion-contaminated materials are potential sources of infection. However, recent studies on the detection of pregnancy-related prion infectivity imply the potential transmission of CWD from mother to offspring. In this review, fundamental aspects of CWD are reviewed.
\end{abstract}

\section{History of chronic wasting disease (CWD) and its current status}

Chronic wasting disease (CWD) is a fatal neurodegenerative prion disease that affects cervids. CWD is transmitted between a range of cervid species such as mule deer (Odocoileus hemionus hemionus), black-tailed deer (Odocoileus hemionus columbianus), white-tailed deer (Odocoileus virginianus), Rocky Mountain elk (Cervus elaphus nelsoni), red deer (Cervus elaphus elaphus), elk (Cervus Canadensis), reindeer (Rangifer tarandus), and Shira's moose (Alces alces shirasi), Reeves' muntjac deer (Muntiacus reevesi) (Williams et al., 1980, 1982; Baeten et al., 2007; Nalls et al., 2013; Haley et al., 2015; Benestad et al., 2016).

The first report of CWD described a wasting disorder in mule deer caught for nutrition 
research near Fort Collins, Colorado in 1967 (Williams et al., 1980). In 1978, pathologists Elizabeth Williams and Stewart Young established that CWD is a form of spongiform encephalopathy (Williams et al., 1980). It was subsequently discovered that CWD not only results in typical vacuolation in neurons (Williams et al., 1980) but also prion protein (PrP) accumulation (Spraker et al., 2002) and infectivity in the brain (Browning et al., 2004).

In 1981, a captive CWD-infected elk was found in Colorado, and then in 1985 a captive CWD-infected mule deer was also identified. Increased surveillance led to the discovery of CWD in Wyoming. To date, the disease has been reported in 24 American states, two Canadian provinces, and South Korea (National Wildlife Health Center, 2018).

The first case of CWD to be detected in Europe (Norway) was in wild Norwegian reindeer (Benestad et al., 2016). In March 2018, Finland also recorded a case of CWD in a wild moose (Finnish Broadcasting Company, 2018). The disease identified in reindeer from Europe is similar to that of CWD in North America. However, CWD of moose in Europe has some distinct features, suggesting a new CWD strain that is different from the CWD strain in North America (EFSA, 2017).

\section{Susceptibility and environmental reservoirs of CWD}

The prevalence of CWD is increasing and is currently present in $>30 \%$ of some free-ranging herds (Edmunds et al., 2016) and more than $80 \%$ of captive herds (Keane et al., 2008b).

The origins of the disease remain unclear. Saprovores, such as buzzard and jackal, and other animals, such as mountain lion, fox, raccoon, coyote and eagle, prey on deer and elk. Thus, CWD may potentially spread widely among a variety of animals. Apart from cervids, CWD-susceptible animals include ferret (Bartz et al., 1998), raccoon (Hamir et al., 2003), squirrel monkey (Marsh et al., 2005), cattle (Hamir et al., 2005, 2007), sheep (Williams, 2005; Hamir et al., 2006), goat (Williams et al., 1992), hamsters (Bartz et al., 1998; Raymond et al., 2007), bank voles (Heisey et al., 2010; Di Bari et al., 2013), mink (Harrington et al., 2008), meadow voles (Heisey et al., 2010), red backed voles (Heisey et al., 2010), white-footed mice (Heisey et al., 2010), deer mice (Heisey et al., 2010) and cats (Mathiason et al., 2013).

The stability of prions in the environment remains unclear at the present time. For example, the concentration of prions in seawater and rivers is unknown. It has been demonstrated that clay components of soil bind to CWD prions, which may contribute to the environmental stability of the bound prion particles (Wyckoff et al., 2016). Indeed, naturally contaminated soil was shown to contain infectious CWD prions that could be experimentally transmitted to elk PrP-expressing transgenic mice (Wyckoff et al., 2016). Surprisingly, soil-bound prions have enhanced infectivity via the oral route compared to unbound prions (Johnson et al., 2007). In addition, using serial protein misfolding cyclic amplification (PMCA), very low levels of proteinase $\mathrm{K}(\mathrm{PK})$-resistant cervid PrP (i.e. an abnormal isoform of cervid $\mathrm{PrP}, \mathrm{PrP}^{\mathrm{CWD}}$ ) were detected in environmental water (Nichols et al., 2009) and mineral licks (Plummer et al., 2018) from a CWD endemic area. Moreover, a recent study reported that CWD prions interact with vegetation of prairie and boreal regions (Kuznetsova et al., 2018). These findings suggest that environmental materials, such as soil and water, may be an important reservoir of prion infectivity and a source of CWD infection. Taken together, these observations highlight the importance of minimizing environmental pollution of CWD prions, particularly in soil and water. 


\section{Clinical symptoms and pathogenesis of CWD}

The characteristic clinical symptoms of CWD-infected animals are slow progressive neurological dysfunction, weight loss, behavioural changes, emaciation, excessive salivation, teeth grinding, fever, anorexia, polyposia, dysphagia, excessive urination, impaired motor coordination and respiratory distress (Sohn et al., 2002; Williams, 2005; Haley et al., 2015). Because these symptoms are not CWD-specific, additional biochemical and pathological analysis is required to verify the diagnosis. Although CWD-infected deer displaying symptoms of the disease can be found throughout the year, infected carcasses are most common in the winter period. The relative profusion of infected carcasses at this time of the year is probably due to the severe winter climate in North America. Furthermore, most deer displaying symptoms of CWD are three to four years of age.

After infection with CWD agent, cellular $\operatorname{PrP}\left(\operatorname{PrP}^{\mathrm{C}}\right)$ is converted into $\operatorname{PrP}{ }^{\mathrm{CWD}}$, which accumulates in brain tissue causing neurological symptoms and ultimately death. $\operatorname{PrP}^{\mathrm{CWD}}$ is primarily distributed in the central nervous system (CNS) and lymphatic tissues, including brain, spinal cord, tonsil, lymph node and spleen. Small quantities of $\operatorname{PrP}^{\mathrm{CWD}}$ agent are also found in the heart and eyes of infected deer. In addition, deboned muscle (Angers et al., 2006) and fat (Race et al., 2009) as well as antler velvet (Angers et al., 2009) have proven to be infectious. Prion infectivity was found in the pregnancy microenvironment of cervids, including the uterus, placenta, ovary, and the placentome (Nalls et al., 2017, 2018). Importantly, CWD prions have been detected in body fluids and excreta such as saliva (Haley et al., 2009a), urine (Haley et al., 2009a), faeces (Tamgüney et al., 2009), amniotic fluid (Nalls et al., 2017), cerebrospinal fluid (Nichols et al., 2012) and blood (Mathiason et al., 2006) (Table 9.1), which may be an important source of infection.

Orally infected CWD-brain homogenate of mule deer induces $\operatorname{PrP}^{\mathrm{CWD}}$ accumulation as early as 42 days post infection in intestine-associated lymphatic tissues, such as retropharyngeal lymph nodes, tonsil, Peyer's patch and ileocecal lymph nodes. Therefore, it has been proposed that CWD agent may be transmitted via clinical and pre-clinical animal carcasses as well as from by-products of deer, such as saliva, stools and urine (Miller et al., 2004; Mathiason et al., 2006; Haley et al., 2009a; Tamgüney et al., 2009). More importantly,

Table 9.1 CWD prions in body fluids and excreta

\begin{tabular}{lll}
\hline Sample type & Species & References \\
\hline Saliva & White-tailed deer & Haley et al. (2009a), Mathiason et al. (2009) \\
Saliva & Mule deer & Mathiason et al. (2006, 2009), Tamgüney et al. (2012) \\
Urine & White-tailed deer & Haley et al. (2009a,b) \\
Blood & Mule deer & Mathiason et al. (2006) \\
Blood & White-tailed deer & Mathiason et al. (2009) \\
Amniotic fluid & Muntjac deer & Nalls et al. (2017) \\
Cerebrospinal fluid & Elk & Nichols et al. (2012) \\
Cerebrospinal fluid & White-tailed deer & Haley et al. (2013) \\
Faeces & Mule deer & Tamgüney et al. (2009) \\
Faeces & White-tailed deer & Haley et al. (2009b) \\
\hline
\end{tabular}


skeletal muscle of CWD-infected deer causes CWD in cervid PrP expressing transgenic mice (Angers et al., 2006). This finding indicates that muscle is a potential source of infection, suggesting that the meat of deer must be treated with caution.

At this time it remains unclear how CWD is spread. Natural or anthropogenic movement of animals may play an important role in the dissemination of CWD. Direct contact between animals and indirect transmission via soil and body surfaces are potential mechanisms for the spread of the disease. CWD is thought to be horizontally infected at a high rate among cervids (Miller et al., 2003). Oral transmission of CWD-infected brain homogenate derived from mule deer to elk has been reported, which is further supported by the finding of CWD-infected free-ranging moose (Baeten et al., 2007). Transfer of infectious material in saliva and/or stools is currently considered a likely means of transmission. The movement of animal carcasses also seems to be involved in the dispersion of CWD. This proposal is supported by evidence that an infected carcass placed in a field resulted in the transmission of CWD to experimental mule deer (Miller et al., 2004), suggesting the spread of CWD may result from a contaminated CWD environment. Recent research has detected the peripheral accumulation of prions and the shedding of infectious prions in bodily fluids, which can be detected even before the appearance of clinical signs (Haley et al., 2017). These findings suggest that not only clinical but also preclinical animals as well as carcasses and by-products may contribute to the transmission and spread of CWD. There is also evidence for the horizontal transmission of CWD based on epidemiological data as well as experimental model studies using transgenic mice expressing the normal cervid prion protein (Seelig et al., 2010).

\section{CWD strains}

Currently, the number of CWD strains and their prevalence, as well as transmission properties, remain unclear. Recent studies indicate the presence of conformational variants or strains of CWD prions may exist. Indeed, sequential passage of a single mule deer CWD isolate using hamster or hamster-PrP expressing transgenic mice causes two distinct disease phenotypes (Raymond et al., 2007; Heisey et al., 2010). Moreover, LaFauci et al. (2006) have reported that elk PrP-expressing transgenic mice developed phenotypically divergent diseases when inoculated with either mule deer or elk CWD, which was suggestive of different strains among cervids. These studies, together with other bioassay data, suggest the presence of predominant strain types comprising at least two, and possibly three, distinct CWD strains in North America (e.g. CWD1 and CWD2; Sgha CWDmd-f and Sgha CWDmd-s; CWD-WI and CWD-CSU; WST and CKY) (Raymond et al., 2007; Angers et al., 2010; Bessen et al., 2011; Perrott et al., 2012; Crowell et al., 2015; Triscott et al., 2015; EFSA, 2017). However, the newly discovered CWD prion in Norway seems to be different from the above-mentioned CWD prion strains (Bian et al., 2018).

\section{Relationship between CWD and human prion diseases}

Several animal prion diseases, such as scrapie, bovine spongiform encephalopathy (BSE), transmissible mink encephalopathy, feline spongiform encephalopathy, and ungulate spongiform encephalopathy, display similar symptoms to CWD. However, there is no known relationship between CWD and these animal prion diseases. In addition, epidemiological 
studies showed no correlation between CWD and human prion diseases (Belay et al., 2004; Anderson et al., 2007; Oszowy et al., 2014; Haley et al., 2015). Most laboratory studies also suggest that the risk of CWD transmission to humans is low. This conclusion is supported by a study in which intracerebral inoculation of CWD prions to transgenic mice expressing human PrP did not lead to the development of any hallmark symptoms of CWD after $>650$ days, while an equivalent inoculation to transgenic mice expressing cervid PrP resulted in infection within 150 days (Kong et al., 2005). These findings indicate the presence of a barrier that makes interspecies transmission unlikely, especially between species as diverse as humans and deer (Kong et al., 2005). Moreover, an in vitro assay showed $\mathrm{PrP}^{\mathrm{CWD}}$ mediated conversion of human PrP to a protease-resistant form is very inefficient, suggesting a species barrier at the molecular level that limits the susceptibility of humans to CWD (Raymond et al., 2000).

In marked contrast, transmission experiments involving non-human primates have shown mixed results. For example, squirrel monkeys were found to be susceptible to CWD infection (Race et al., 2014), although there was no clinical, pathological, or biochemical evidence of CWD transmission to cynomolgus macaques (Race et al., 2018). Currently, it is unclear why cynomolgus macaques and squirrel monkeys give markedly different results with regard to CWD transmission. Although the conclusions to be drawn from studies using non-human primates as infection models are still a matter of debate, a plethora of laboratory experiments suggest the risk of CWD transmission to humans is low (Hannaoui et al., 2017). Nonetheless, the potential zoonotic transmission of CWD is an alarming prospect. Given that the scientific information on CWD is limited, consumption of CWD-positive animals should be prohibited at this time.

\section{Diagnostic methods for CWD}

The gold standard of CWD diagnosis is immunohistochemistry (IHC) (Haley et al., 2017). Medullary obex and lymphatic tissues, such as tonsil and pharyngeal lymph nodes, are used for this analysis. Dorsal motor nucleus of the vagus (DMNV) in the obex region of the brainstem and the medial retropharyngeal lymph nodes (RLN) is generally used for regulatory diagnosis (Sigurdson et al., 1999; Peters et al., 2000; Keane et al., 2008a). IHC is a method of observing tissue sections stained with anti-PrP antibody using light microscopy. This methodology has merits in terms of its high level of sensitivity and specificity. In addition, IHC can be used to verify whether the tissue distribution of $\mathrm{PrP}^{\mathrm{CWD}}$ is as anticipated. Other methods for CWD diagnosis include enzyme-linked immunosorbent assay (ELISA) and western blotting. ELISA is used to detect PrP adsorbed on plates after treatment of brain homogenate with PK. Western blotting detects PrP absorbed on a membrane after an electroblotting procedure. ELISA is preferable because the technique allows rapid and quantitative analysis. However, western blotting facilitates more detailed biochemical analysis such as determination of the molecular weight of PrP. Such information may be used to help identify the prion source and specific strain.

The United States Department of Agriculture (USDA) has recently certified an ELISA kit as an official screening test for CWD using obex and lymph node tissues (USDA, 2017). Further developments in detecting CWD have enabled antemortem diagnosis using IHC of tonsil tissue. In addition, a method for prion diagnosis, known as PMCA, has been developed that amplifies PK-resistant PrP by sequential incubation and sonication using a mixture of 
$\operatorname{PrP}^{\mathrm{C}}$ source and $\mathrm{PrP}^{\mathrm{CWD}}$ seed. PMCA can detect $\mathrm{PrP}^{\mathrm{CWD}}$ in the urine of presymptomatic deer (Rubenstein et al., 2011) and the blood of asymptomatic deer (Kramm et al., 2017).

More recently, a real-time quaking-induced conversion (RT-QuIC) assay, which is a modified version of PMCA for enabling the quantification of $\mathrm{PrP}^{\mathrm{CWD}}$ at high sensitivity, has been developed (Manne et al., 2017). Using this methodology it is possible to perform antemortem detection of CWD in recto-anal mucosa-associated lymphoid tissue (RAMALT). The performance of RT-QuIC showed good results at $92 \%$ relative sensitivity and $95 \%$ relative specificity using blinded samples compared to IHC results. Other researchers also detected PrP ${ }^{C W D}$ from RAMALT and nasal brushings using RT-QuIC (Haley et al., 2016).

Powerful research tools to analyse CWD will be invaluable in helping to reveal the molecular basis of the disease. For example, a transgenic mouse expressing cervid PrP (Browning et al., 2004; Seelig et al., 2010) and a CWD-susceptible cell line (Raymond et al., 2006) have been developed. Such tools will be used to devise bioassays of CWD infectivity in tissues and body fluids derived from CWD-infected cervids.

Taken together, the recent development of diagnostic technologies, such as qualitative and quantitative amplification techniques, enables not only postmortem but also antemortem detection of $\mathrm{PrP}^{\mathrm{CWD}}$ from peripheral tissues and body fluids. In addition, these diagnostic methods will contribute to further risk assessment of deer tissues and body fluids obtained from CWD-endemic areas as well as improving surveillance programs.

\section{Conclusion and perspectives}

There is a strikingly high incidence of CWD in wild animals, such as deer and elk, which maintains a reservoir of CWD prions that is difficult to counteract by the simple approach of targeted culling. In addition, the high stability of CWD prions in the environment further exacerbates the problem. Moreover, the diverse distribution of CWD prions within the body of infected cervids, including neurological and peripheral tissues as well as muscle, fat and pregnancy tissues, increases the chances of transmitting the disease. In particular, the presence of CWD prions in body fluids, such as saliva, faeces and urine, enhances infectivity and may lead to both horizontal and vertical transmission. Although more than two CWD strains have been found, their host range and prevalence as well as transmission features remain unclear at the present time.

To date, laboratory studies and epidemiological investigations suggest little association between human prion diseases and exposure to CWD prions. However, it is not known whether there is an absolute species barrier for humans against all strains of CWD prions. Thus, long-term investigations to study potential human infection and exposure to CWD prions are needed.

\section{References}

Anderson, C.A., Bosque, P., Filley, C.M., Arciniegas, D.B., Kleinschmidt-Demasters, B.K., Pape, W.J., and Tyler, K.L. (2007). Colorado surveillance program for chronic wasting disease transmission to humans: lessons from 2 highly suspicious but negative cases. Arch. Neurol. 64, 439-441.

Angers, R.C., Browning, S.R., Seward, T.S., Sigurdson, C.J., Miller, M.W., Hoover, E.A., and Telling, G.C. (2006). Prions in skeletal muscles of deer with chronic wasting disease. Science 311, 1117.

Angers, R.C., Kang, H.E., Napier, D., Browning, S., Seward, T., Mathiason, C., Balachandran, A., McKenzie,

D., Castilla, J., Soto, C., et al. (2010). Prion strain mutation determined by prion protein conformational compatibility and primary structure. Science 328, 1154-8. https://doi.org/10.1126/science.1187107 
Angers, R.C., Seward, T.S., Napier, D., Green, M., Hoover, E., Spraker, T., O’Rourke, K., Balachandran, A., and Telling, G.C. (2009). Chronic wasting disease prions in elk antler velvet. Emerging Infect. Dis. 15, 696-703. https://doi.org/10.3201/eid1505.081458

Baeten, L.A., Powers, B.E., Jewell, J.E., Spraker, T.R., and Miller, M.W. (2007). A natural case of chronic wasting disease in a free-ranging moose (Alces alces shirasi). J. Wildl. Dis. 43, 309-314.

Belay, E.D., Maddox, R.A., Williams, E.S., Miller, M.W., Gambetti, P., and Schonberger, L.B. (2004). Chronic wasting disease and potential transmission to humans. Emerging Infect. Dis. 10, 977-984.

Bartz, J.C., Marsh, R.F., McKenzie, D.I., and Aiken, J.M. (1998). The host range of chronic wasting disease is altered on passage in ferrets. Virology 251, 297-301.

Benestad, S.L., Mitchell, G., Simmons, M., Ytrehus, B., and Vikøren, T. (2016). First case of chronic wasting disease in Europe in a Norwegian free-ranging reindeer. Vet. Res. 47, 88. https://doi.org/10.1186/ s13567-016-0375-4

Bessen, R.A., Robinson, C.J., Seelig, D.M., Watschke, C.P., Lowe, D., Shearin, H., Martinka, S., and Babcock, A.M. (2011). Transmission of chronic wasting disease identifies a prion strain causing cachexia and heart infection in hamsters. PLOS ONE 6, e28026. https://doi.org/10.1371/journal.pone.0028026

Bian, J., Moreno, J.A., Kim, S., Vikoren, T., Madslien, K., Vage, J., Benestad, S.L., and Telling, G.C. (2018). The strain properties of prions causing chronic wasting disease in Norwegian cervids are distinct from those causing disease in North America, Prion 07, 6.

Browning, S.R., Mason, G.L., Seward, T., Green, M., Eliason, G.A., Mathiason, C., Miller, M.W., Williams, E.S., Hoover, E., and Telling, G.C. (2004). Transmission of prions from mule deer and elk with chronic wasting disease to transgenic mice expressing cervid PrP. J. Virol. 78, 13345-13350.

Crowell, J., Hughson, A., Caughey, B., and Bessen, R.A. (2015). Host determinants of prion strain diversity independent of prion protein genotype. J. Virol. 89, 10427-41. https://doi.org/10.1128/JVI.01586-15

Di Bari, M.A., Nonno, R., Castilla, J., D’Agostino, C., Pirisinu, L., Riccardi, G., Conte, M., Richt, J., Kunkle, R., Langeveld, J., et al. (2013). Chronic wasting disease in bank voles: characterisation of the shortest incubation time model for prion diseases. PLOS Pathog. 9, e1003219. https://doi.org/10.1371/ journal.ppat.1003219

Edmunds, D.R., Kauffman, M.J., Schumaker, B.A., Lindzey, F.G., Cook, W.E., Kreeger, T.J., Grogan, R.G., and Cornish, T.E. (2016). Chronic wasting disease drives population decline of white-tailed deer. PLOS ONE. 11, e0161127. https://doi.org/10.1371/journal.pone.0161127

EFSA Panel on Biological Hazards (BIOHAZ), Ricci, A., Allende, A., Bolton, D., Chemaly, M., Davies, R., Escámez, P.S.F., Gironés, R., Herman, L., Koutsoumanis, K., et al. (2017). Chronic wasting disease (CWD) in cervids. EFSA J. 15, 4667.

Finnish Broadcasting Company (Yleisradio Oy). 2018. First case in Finland: Elk dies due to chronic wasting disease. Available online: https://yle.fi/uutiset/osasto/news/first_case_in_finland_elk_ dies_due_to_chronic_wasting_disease/10108115. Accessed 21 June 2018.

Haley, N.J., Seelig, D.M., Zäbel, M.D., Telling, G.C., and Hoover, E.A. (2009). Detection of CWD prions in urine and saliva of deer by transgenic mouse bioassay. PLOS ONE 4, e4848. https://doi.org/10.1371/ journal.pone.0004848

Haley, N.J., Mathiason, C.K., Zabel, M.D., Telling, G.C., and Hoover, E.A. (2009). Detection of sub-clinical CWD infection in conventional test-negative deer long after oral exposure to urine and feces from CWD+ deer. PLOS ONE 4, e7990. https://doi.org/10.1371/journal.pone.0007990

Haley, N.J., Van de Motter, A., Carver, S., Henderson, D., Davenport, K., Seelig, D.M., Mathiason, C., and Hoover, E. (2013). Prion-seeding activity in cerebrospinal fluid of deer with chronic wasting disease. PLOS ONE 8, e81488. https://doi.org/10.1371/journal.pone.0081488

Haley, N.J., and Hoover, E.A. (2015). Chronic wasting disease of cervids: current knowledge and future perspectives. Annu. Rev. Anim. Biosci. 3, 305-25. https://doi.org/10.1146/annurevanimal-022114-111001

Haley, N.J., Siepker, C., Walter, W.D., Thomsen, B.V., Greenlee, J.J., Lehmkuhl, A.D., and Richt, J.A. (2016). Antemortem detection of chronic wasting disease prions in nasal brush collections and rectal biopsy specimens from white-tailed deer by real-time quaking-induced conversion. J. Clin. Microbiol. 54, 1108-16. https://doi.org/10.1128/JCM.02699-15

Haley, N.J., and Richt, J.A. (2017). Evolution of diagnostic tests for chronic wasting disease, a naturally occurring prion disease of cervids. Pathogens 6, 35.

Hamir, A.N., Miller, J.M., Cutlip, R.C., Stack, M.J., Chaplin, M.J., Jenny, A.L., and Williams, E.S. (2003). Experimental inoculation of scrapie and chronic wasting disease agents in raccoons (Procyon lotor). Vet. Rec. $153,121-123$. 
Hamir, A.N., Kunkle, R.A., Cutlip, R.C., Miller, J.M., O’Rourke, K.I., Williams, E.S., Miller, M.W., Stack, M.J., Chaplin, M.J., and Richt, J.A. (2005). Experimental transmission of chronic wasting disease agent from mule deer to cattle by the intracerebral route. J. Vet. Diagn. Invest. 17, 276-81. https://doi. org $/ 10.1177 / 104063870501700313$

Hamir, A.N., Kunkle, R.A., Cutlip, R.C., Miller, J.M., Williams, E.S., and Richt, J.A. (2006). Transmission of chronic wasting disease of mule deer to Suffolk sheep following intracerebral inoculation. J. Vet. Diagn. Invest. 18, 558-65. https://doi.org/10.1177/104063870601800606

Hamir, A.N., Miller, J.M., Kunkle, R.A., Hall, S.M., and Richt, J.A. (2007). Susceptibility of cattle to first-passage intracerebral inoculation with chronic wasting disease agent from white-tailed deer. Vet. Pathol. 44, 487-493.

Hannaoui, S., Schatzl, H.M., and Gilch, S. (2017). Chronic wasting disease: Emerging prions and their potential risk. PLOS Pathog. 13, e1006619. https://doi.org/10.1371/journal.ppat.1006619

Harrington, R.D., Baszler, T.V., O’Rourke, K.I., Schneider, D.A., Spraker, T.R., Liggitt, H.D., and Knowles, D.P. (2008). A species barrier limits transmission of chronic wasting disease to mink (Mustela vison). J. Gen. Virol. 89, 1086-1096.

Heisey, D.M., Mickelsen, N.A., Schneider, J.R., Johnson, C.J., Johnson, C.J., Langenberg, J.A., Bochsler, P.N., Keane, D.P., and Barr, D.J. (2010). Chronic wasting disease (CWD) susceptibility of several North American rodents that are sympatric with cervid CWD epidemics. J. Virol. 84, 210-5. https://doi. org/10.1128/JVI.00560-09

Johnson, D., White,J.,Lim, L., and Davison, A.N. (1975). The effect of experimental hyperphenylalaninaemia on polyribosomes of the developing rat brain. Biochem. Soc. Trans. 3, 93-94.

Keane, D.P., Barr, D.J., Keller, J.E., Hall, S.M., Langenberg, J.A., and Bochsler, P.N. (2008a). Comparison of retropharyngeal lymph node and obex region of the brainstem in detection of chronic wasting disease in white-tailed deer (Odocoileus virginianus). J. Vet. Diagn. Invest. 20, 58-60.

Keane, D.P., Barr, D.J., Bochsler, P.N., Hall, S.M., Gidlewski, T., O’Rourke, K.I., Spraker, T.R., and Samuel, M.D. (2008b). Chronic wasting disease in a Wisconsin white-tailed deer farm. J. Vet. Diagn. Invest. 20, 698-703.

Kong, Q. Huang, S., Zou, W., Vanegas, D., Wang, M., Wu, D., Yuan, J., Zheng, M., Bai, H., Deng, H., et al. (2005). Chronic wasting disease of elk: transmissibility to humans examined by transgenic mouse models. J. Neurosci. 25, 7944-7949.

Kramm, C., Pritzkow, S., Lyon, A., Nichols, T., Morales, R., and Soto, C. (2017). Detection of prions in blood of cervids at the asymptomatic stage of chronic wasting disease. Sci. Rep. 7, 17241. https://doi. org/10.1038/s41598-017-17090-x

Kuznetsova, A., Triscott, E., McKenzie, D., and Aiken, J.M. (2018). Interaction of CWD prions with environmental samples. Prion $W A 7,35$.

LaFauci, G., Carp, R.I., Meeker, H.C., Ye, X., Kim, J.I., Natelli, M., Cedeno, M., Petersen, R.B., Kascsak, R., and Rubenstein, R. (2006). Passage of chronic wasting disease prion into transgenic mice expressing Rocky Mountain elk (Cervus elaphus nelsoni) PrPC. J. Gen. Virol. 87, 3773-3780.

Manne, S., Kondru, N., Nichols, T., Lehmkuhl, A., Thomsen, B., Main, R., Halbur, P., Dutta, S., and Kanthasamy, A.G. (2017). Ante-mortem detection of chronic wasting disease in recto-anal mucosa-associated lymphoid tissues from elk (Cervus elaphus nelsoni) using real-time quaking-induced conversion (RT-QuIC) assay: A blinded collaborative study. Prion 11, 415-430. https://doi.org/10.1 080/19336896.2017.1368936

Marsh, R.F., Kincaid, A.E., Bessen, R.A., and Bartz, J.C. (2005). Interspecies transmission of chronic wasting disease prions to squirrel monkeys (Saimiri sciureus). J. Virol. 79, 13794-13796.

Mathiason, C.K., Powers, J.G., Dahmes, S.J., Osborn, D.A., Miller, K.V., Warren, R.J., Mason, G.L., Hays, S.A., Hayes-Klug, J., Seelig, D.M., et al. (2006). Infectious prions in the saliva and blood of deer with chronic wasting disease. Science 314, 133-136.

Mathiason, C.K., Hays, S.A., Powers, J., Hayes-Klug, J., Langenberg, J., Dahmes, S.J., Osborn, D.A., Miller, K.V., Warren, R.J., Mason, G.L., et al. (2009). Infectious prions in pre-clinical deer and transmission of chronic wasting disease solely by environmental exposure. PLOS ONE 4, e5916. https://doi. org/10.1371/journal.pone.0005916

Mathiason, C.K., Nalls, A.V., Seelig, D.M., Kraft, S.L., Carnes, K., Anderson, K.R., Hayes-Klug, J., and Hoover, E.A. (2013). Susceptibility of domestic cats to chronic wasting disease. J. Virol. 87, 1947-56. https://doi.org/10.1128/JVI.02592-12

Miller, M.W., and Williams, E.S. (2003). Prion disease: horizontal prion transmission in mule deer. Nature 425, 35-6. https://doi.org/10.1038/425035a 
Miller, M.W., Williams, E.S., Hobbs, N.T., and Wolfe, L.L. (2004). Environmental sources of prion transmission in mule deer. Emerging Infect. Dis. 10, 1003-1006.

Nalls, A.V., McNulty, E., Powers, J., Seelig, D.M., Hoover, C., Haley, N.J., Hayes-Klug, J., Anderson, K., Stewart, P., Goldmann, W., et al. (2013). Mother to offspring transmission of chronic wasting disease in Reeves' muntjac deer. PLOS ONE 8, e71844. https://doi.org/10.1371/journal.pone.0071844

Nalls, A.V., McNulty, E., Hoover, C.E., Pulscher, L.A., Hoover, E.A., and Mathiason, C.K. (2017). Infectious prions in the pregnancy microenvironment of chronic wasting disease-infected Reeves' muntjac deer. J. Virol. 91, pii: e00501-17

Nalls, A.V., McNulty, E.E., Hoover, C., Hoover, E.A., Wild, M., Powers, J., Selariu, A., and Mathiason, C.K. (2018). An overview-chronic wasting disease mother to offspring transmission studies conducted at Colorado state university. Prion WA1, 32.

National Wildlife Health Center. (2018). Available online: https:www.usgs.gov/centers/nwhc. Accessed 13 October 2018.

Nichols, T.A., Pulford, B., Wyckoff, A.C., Meyerett, C., Michel, B., Gertig, K., Hoover, E.A., Jewell, J.E., Telling, G.C., and Zabel, M.D. (2009). Detection of protease-resistant cervid prion protein in water from a CWD-endemic area. Prion 3, 171-183.

Nichols, T.A., Spraker, T.R., Gidlewski, T., Powers, J.G., Telling, G.C., VerCauteren, K.C., and Zabel, M.D. (2012). Detection of prion protein in the cerebrospinal fluid of elk (Cervus canadensis nelsoni) with chronic wasting disease using protein misfolding cyclic amplification. J. Vet. Diagn. Invest. 24, 746-9. https://doi.org/10.1177/1040638712448060

Olszowy, K.M., Lavelle, J., Rachfal, K., Hempstead, S., Drouin, K., Darcy, J.M., Reiber, C., and Garruto, R.M. (2014). Six-year follow-up of a point-source exposure to CWD contaminated venison in an Upstate New York community: risk behaviours and health outcomes 2005-2011. Public Health 128, 860-8. https://doi.org/10.1016/j.puhe.2014.06.012

Perrott, M.R., Sigurdson, C.J., Mason, G.L., and Hoover, E.A. (2012). Evidence for distinct chronic wasting disease (CWD) strains in experimental CWD in ferrets. J. Gen. Virol. 93, 212-221.

Peters, J., Miller, J.M., Jenny, A.L., Peterson, T.L., and Carmichael, K.P. (2000). Immunohistochemical diagnosis of chronic wasting disease in preclinically affected elk from a captive herd. J. Vet. Diagn. Invest. 12, 579-82. https://doi.org/10.1177/104063870001200618

Plummer, I.H., Johnson, C.J., Chesney, A.R., Pedersen, J.A., and Samuel, M.D. (2018). Mineral licks as environmental reservoirs of chronic wasting disease prions. PLOS ONE 13, e0196745. https://doi. org/10.1371/journal.pone.0196745

Race, B., Meade-White, K., Race, R., and Chesebro, B. (2009). Prion infectivity in fat of deer with chronic wasting disease. J. Virol. 83, 9608-10. https://doi.org/10.1128/JVI.01127-09

Race, B., Meade-White, K.D., Phillips, K., Striebel, J., Race, R., and Chesebro, B. (2014). Chronic wasting disease agents in nonhuman primates. Emerging Infect. Dis. 20, 833-7. https://doi.org/10.3201/ eid2005.130778

Race, B., Williams, K., Orrú, C.D., Hughson, A.G., Lubke, L., and Chesebro, B. (2018). Lack of transmission of chronic wasting disease to cynomolgus macaques. J. Virol. [Epub ahead of print].

Raymond, G.J., Bossers, A., Raymond, L.D., O’Rourke, K.I., McHolland, L.E., Bryant, P.K., Miller, M.W., Williams, E.S., Smits, M., and Caughey, B. (2000). Evidence of a molecular barrier limiting susceptibility of humans, cattle and sheep to chronic wasting disease. EMBO J. 19, 4425-30. https:// doi.org/10.1093/emboj/19.17.4425

Raymond, G.J., Olsen, E.A., Lee, K.S., Raymond, L.D., Bryant, P.K., Baron, G.S., Caughey, W.S., Kocisko, D.A., McHolland, L.E., Favara, C., et al. (2006). Inhibition of protease-resistant prion protein formation in a transformed deer cell line infected with chronic wasting disease. J. Virol. 80, 596-604.

Raymond, G.J., Raymond, L.D., Meade-White, K.D., Hughson, A.G., Favara, C., Gardner, D., Williams, E.S., Miller, M.W., Race, R.E., and Caughey, B. (2007). Transmission and adaptation of chronic wasting disease to hamsters and transgenic mice: evidence for strains. J. Virol. 81, 4305-4314.

Rubenstein, R., Chang, B., Gray, P., Piltch, M., Bulgin, M.S., Sorensen-Melson, S., and Miller, M.W. (2011). Prion disease detection, PMCA kinetics, and IgG in urine from sheep naturally/experimentally infected with scrapie and deer with preclinical/clinical chronic wasting disease. J. Virol. 85, 9031-8. https://doi. org/10.1128/JVI.05111-11

Seelig, D.M., Mason, G.L., Telling, G.C., and Hoover, E.A. (2010). Pathogenesis of chronic wasting disease in cervidized transgenic mice. Am. J. Pathol. 176, 2785-97. https://doi.org/10.2353/ajpath.2010.090710

Sigurdson, C.J., Williams, E.S., Miller, M.W., Spraker, T.R., O’Rourke, K.I., and Hoover, E.A. (1999). Oral transmission and early lymphoid tropism of chronic wasting disease PrPres in mule deer fawns (Odocoileus hemionus). J. Gen. Virol. 80, 2757-64. https://doi.org/10.1099/0022-1317-80-10-2757 
Sohn, H.J., Kim, J.H., Choi, K.S., Nah, J.J., Joo, Y.S., Jean, Y.H., Ahn, S.W., Kim, O.K., Kim, D.Y., and Balachandran, A. (2002). A case of chronic wasting disease in an elk imported to Korea from Canada. J. Vet. Med. Sci. 64, 855-858.

Spraker, T.R., Zink, R.R., Cummings, B.A., Wild, M.A., Miller, M.W., and O’Rourke, K.I. (2002). Comparison of histological lesions and immunohistochemical staining of proteinase-resistant prion protein in a naturally occurring spongiform encephalopathy of free-ranging mule deer (Odocoileus hemionus) with those of chronic wasting disease of captive mule deer. Vet. Pathol. 39, 110119.

Tamgüney, G., Miller, M.W., Wolfe, L.L., Sirochman, T.M., Glidden, D.V., Palmer, C., Lemus, A., DeArmond, S.J., and Prusiner, S.B. (2009). Asymptomatic deer excrete infectious prions in faeces. Nature 461, 529-32. https://doi.org/10.1038/nature08289

Tamgüney, G., Richt, J.A., Hamir, A.N., Greenlee, J.J., Miller, M.W., Wolfe, L.L., Sirochman, T.M., Young, A.J., Glidden, D.V., Johnson, N.L., et al. (2012). Salivary prions in sheep and deer. Prion 6, 52-61. https://doi.org/10.4161/pri.6.1.16984

Triscott, E., Duque-Velasquez, C., Aiken, J.M., and McKenzie, D. (2015). Chronic wasting disease (CWD) transmission into hamsters. Prion. 9, S86.

USDA. (2017). CWD Diagnostics. Available online: https://www.aphis.usda.gov/aphis/ourfocus/ animalhealth/animal-disease-information/sa_alternate_livestock/sa_cervid_health/sa_cwd/ ct_diagnostics. Accessed 21 June 2018.

Williams, E.S., and Young, S. (1980). Chronic wasting disease of captive mule deer: a spongiform encephalopathy. J. Wildl. Dis. 16, 89-98.

Williams, E.S., and Young, S. (1982). Spongiform encephalopathy of Rocky Mountain elk. J. Wildl. Dis. $18,465-471$.

Williams, E.S., and Young, S. (1992). Spongiform encephalopathies in Cervidae. Rev. Sci. Tech. 11, 551-567.

Williams, E.S. (2005). Chronic wasting disease. Vet. Pathol. 42, 530-549.

Wyckoff, A.C., Kane, S., Lockwood, K., Seligman, J., Michel, B., Hill, D., Ortega, A., Mangalea, M.R., Telling, G.C., Miller, M.W., et al. (2016). Clay components in soil dictate environmental stability and bioavailability of cervid prions in mice. Front. Microbiol. 7, 1885. https://doi.org/10.3389/ fmicb.2016.01885 